\title{
Probabilistic versus deterministic tractography for delineation of the cortico-subthalamic hyperdirect pathway in patients with Parkinson disease selected for deep brain stimulation
}

\author{
Mikkel V. Petersen, MD, ${ }^{1,2}$ Torben E. Lund, MSc, PhD, ${ }^{1}$ Niels Sunde, MD, PhD, ${ }^{2}$ \\ Jesper Frandsen, MSc, ${ }^{1}$ Frederikke Rosendal, MD, PhD, ${ }^{2}$ Niels Juul, MD, ${ }^{3}$ and \\ Karen Østergaard, MD, PhD ${ }^{4}$ \\ ${ }^{1}$ Center of Functionally Integrative Neuroscience and Departments of ${ }^{2}$ Neurosurgery, ${ }^{3}$ Neuroanaesthesiology, and ${ }^{4}$ Neurology, \\ Aarhus University Hospital, Aarhus, Denmark
}

OBJECTIVE Diffusion-weighted MRI (DWI) and tractography allows noninvasive mapping of the structural connections of the brain, and may provide important information for neurosurgical planning. The hyperdirect pathway, connecting the subthalamic nucleus (STN) with the motor cortex, is assumed to play a key role in mediating the effects of deep brain stimulation (DBS), which is an effective but poorly understood treatment for Parkinson disease. This study aimed to apply recent methodological advances in DWI acquisition and analysis to the delineation of the hyperdirect pathway in patients with Parkinson disease selected for surgery.

METHODS High spatial and angular resolution DWI data were acquired preoperatively from 5 patients with Parkinson disease undergoing DBS. The authors compared the delineated hyperdirect pathways and associated STN target maps generated by 2 different tractography methods: a tensor-based deterministic method, typically available in clinical settings, and an advanced probabilistic method based on constrained spherical deconvolution. In addition, 10 highresolution data sets with the same scanning parameters were acquired from a healthy control participant to assess the robustness of the tractography results.

RESULTS Both tractography approaches identified connections between the ipsilateral motor cortex and the STN. However, the 2 methods provided substantially different target regions in the STN, with the target center of gravity differing by $>1.4 \mathrm{~mm}$ on average. The probabilistic method (based on constrained spherical deconvolution) plausibly reconstructed a continuous set of connections from the motor cortex, terminating in the dorsolateral region of the STN. In contrast, the tensor-based method reconstructed a comparatively sparser and more variable subset of connections. Furthermore, across the control scans, the probabilistic method identified considerably more consistent targeting regions within the STN compared with the deterministic tensor-based method, which demonstrated a 1.9-2.4 times higher variation.

CONCLUSIONS These data provide a strong impetus for the use of a robust probabilistic tractography framework based on constrained spherical deconvolution, or similar advanced DWI models, in clinical settings. The inherent limitations and demonstrated inaccuracy of the tensor-based method leave it questionable for use in high-precision stereotactic DBS surgery. The authors have also described a straightforward method for importing tractography-derived information into any clinical neuronavigation system, based on the generation of track-density images.

https://thejns.org/doi/abs/10.3171/2016.4.JNS1624

KEY WORDS diffusion-weighted imaging; tractography; deep brain stimulation; constrained spherical deconvolution; Parkinson disease; stereotactic surgery; neuronavigation; functional neurosurgery

\footnotetext{
ABBREVIATIONS BA = Brodmann area; CSD = constrained spherical deconvolution; CST = corticospinal tract; DBS = deep brain stimulation; DT = diffusion tensor; DWI = diffusion-weighted MRI; EPI = echo planar imaging; HDP = hyperdirect pathway; $\mathrm{PD}=$ Parkinson disease; RESOLVE = readout segmentation of long variable echo trains; STN = subthalamic nucleus; TDI = track-density image.
}

SUBMITTED January 4, 2016. ACCEPTED April 29, 2016.

INCLUDE WHEN CITING Published online July 8, 2016; DOI: 10.3171/2016.4.JNS1624. 
$\mathrm{H}$ IGH-FREQUENCY deep brain stimulation (DBS) in the subthalamic nucleus (STN) is an effective treatment for patients who have Parkinson disease (PD) with motor fluctuations and dyskinesia. However, there can be adverse, neurocognitive side effects that only become apparent over time, and negatively impact treatment outcomes in DBS. ${ }^{8,38,48}$ Although the underlying mechanisms are not yet understood, it is clear that suboptimal placement of electrodes, and spread of current to surrounding structures, can lead to both short- and longterm negative side effects. Improved preoperative targeting is one avenue for refining DBS treatment, and in vivo tractography offers a major methodological opportunity to facilitate this. ${ }^{17}$ Successful implementation would represent a substantial advance toward individualized treatment planning and a potential for improvement in the field of functional neurosurgery. In this study, we used a stateof-the-art diffusion-weighted MRI (DWI) acquisition to examine the feasibility and robustness of implementing tractography to target the STN.

Diffusion-based tractography is a rapidly developing neuroimaging technique that uniquely allows noninvasive investigation of the structural networks in the human brain. ${ }^{3}$ Recent advances in both acquisition and processing of DWI data allow us to explore the underlying white matter pathways with greater detail and precision than previously achievable. ${ }^{42}$ Although there has been much excitement about the potential of tractography for studying neural networks and white matter integrity, it is not without limitations, as reviewed extensively elsewhere. ${ }^{19-23}$ Briefly, a major challenge lies in accurately modeling complex fiber configurations in voxels containing multiple crossing-fiber populations. Recent studies indicate that the proportion of white matter voxels containing such crossing fibers is as high as $90 \% .{ }^{19}$ Despite a clear need for more advanced modeling, ${ }^{44}$ the original diffusion-tensor (DT) method, which can only model 1 principal fiber orientation per voxel, still dominates in neurosurgical research. ${ }^{11}$

Numerous higher-order diffusion models using probabilistic tractography techniques, such as the constrained spherical deconvolution (CSD) method implemented in this work, have been developed to model and track through voxels containing multiple fiber orientations. ${ }^{44,45}$ It is generally agreed that these advanced models provide more anatomically plausible tractography results and are necessary to analyze the complexities of the human brain., $311,19,44$ However, these advanced techniques introduce additional requirements for MRI acquisition protocols and subsequent data processing, which is not supported directly by available commercial neuronavigation systems. Despite these technical challenges, tractography may provide invaluable information for neurosurgical planning and is being increasingly investigated for this purpose..$^{12,28}$

The traditional view of STN anatomy and function, as established in primate studies, is that of 3 functionally and anatomically distinct regions (sensorimotor, associative, and limbic). ${ }^{31,37}$ This classic tripartite segmentation has been questioned recently. ${ }^{27}$ Convergent evidence now suggests a topological organization of the STN, without sharply delineated anatomical boundaries and instead a degree of overlap between functional zones. ${ }^{16}$ The men- tioned study also confirmed findings from previous neuronal tracing studies in animals that the primary motor cortex (Brodmann area [BA] 4) and the premotor and supplementary motor cortex (BA6) project directly to the STN, forming a cortico-subthalamic hyperdirect pathway (HDP). ${ }^{33-35}$ Recent optogenetic studies using parkinsonian rodents have provided robust evidence that antidromic activation of this pathway plays an important role in mediating DBS treatment effects. ${ }^{15}$

A small number of studies have previously used advanced probabilistic tractography methods to examine STN connectivity. 1,6,26,32 These studies, performed in healthy volunteers, have been able to identify functional regions within the STN in accordance with known primate anatomy. In addition, there are only a few DTbased tractography studies in patients with PD undergoing DBS. ${ }^{4,49}$ The simpler DT-based tractography method has the practical advantage of being available in a small number of commercial neuronavigation systems, whereas more advanced probabilistic methods are not. The disadvantage of the available DT-based methods is that they have been superseded in the nonclinical neuroimaging literature by more advanced methods, which are clearly

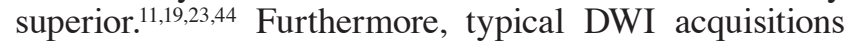
are severely impacted in the STN region by both cardiac pulsation and susceptibility-induced distortions, challenging this specific clinical application.

The main aim of the current study was to extend and evaluate the use of a robust CSD-based probabilistic tractography method into an important clinical setting. We directly compared the impact of using this probabilistic method with a DT-based deterministic approach for delineating and targeting the HDP in a small group of patients with PD selected for DBS treatment. Furthermore, we investigated the consistency of the 2 tractography methods across repeated MRI scans $(\mathrm{N}=10)$ of 1 healthy adult. Finally, we acquired high-quality DWI data for this study by using a recently developed, advanced (navigator echocorrected) readout-segmented echo planar imaging (EPI) sequence. ${ }^{40}$

\section{Methods}

\section{Selection of Participants}

Five patients with PD selected for STN DBS surgery at Aarhus University Hospital participated in the current study (average age 54.8 [SD 5.7] years) and had an average disease duration of 10 years (SD 4 years). All patients showed significant, positive response to levodopa challenge tests (mean improvement of 71\% [SD 9\%]) in Unified Parkinson's Disease Rating Scale Part III score. One patient diagnosed with dopa-responsive dystonia (DYT5), initially with a good treatment effect of botulinum toxin, developed left-sided PD cardinal motor symptoms responsive to levodopa treatment. When the patient experienced off periods and dyskinesia, a dopamine transporterSPECT scan was performed, confirming bilateral putaminal loss of dopaminergic terminals.

All patients underwent several preoperative evaluations by an experienced multidisciplinary team consisting of a neuropsychologist, neurologist, and neurosurgeon 


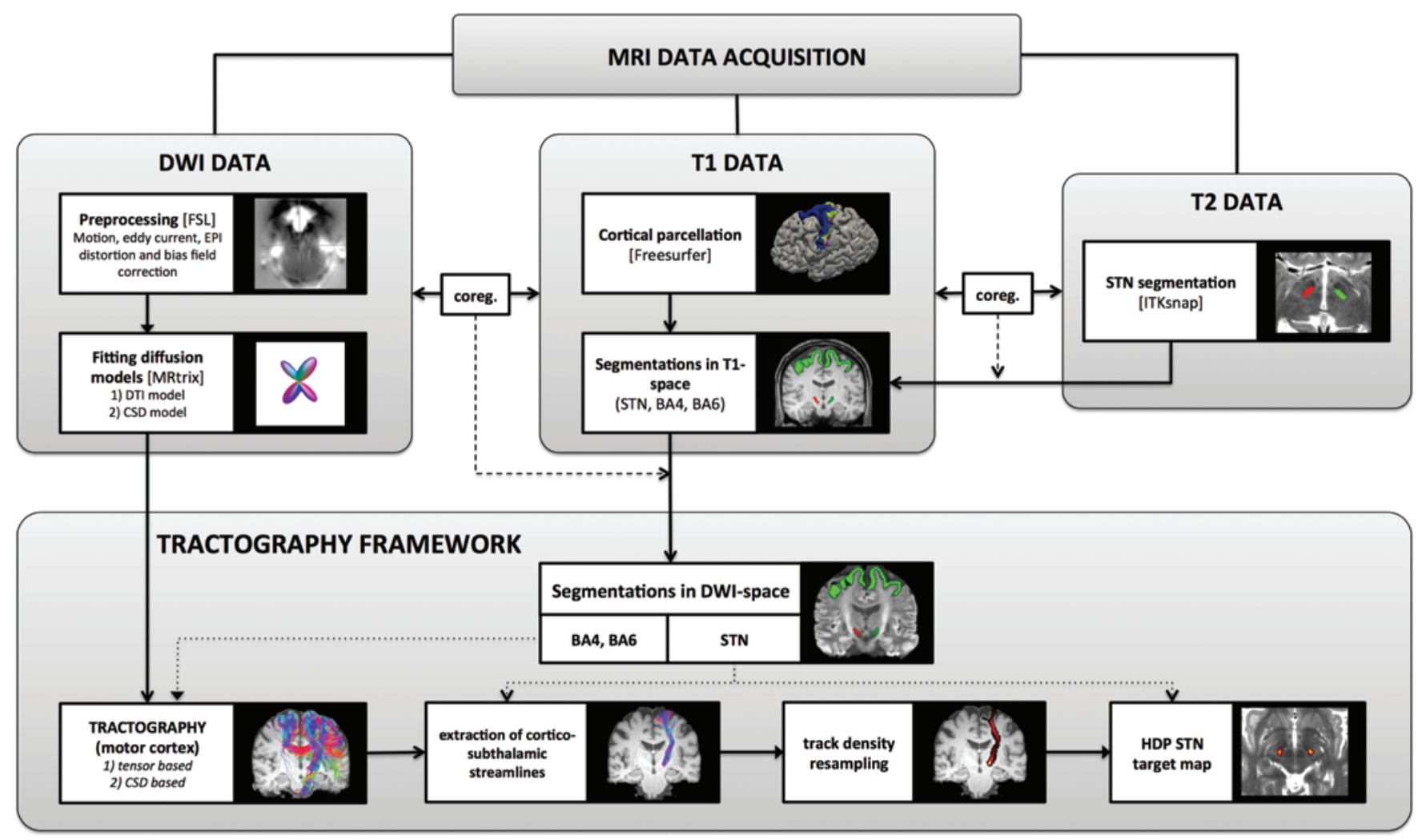

FIG. 1. Flow chart illustrating the processing pipeline. Coreg. = coregistration. Figure is available in color online only.

to ensure DBS candidacy. Patient selection criteria and DBS electrode implantation were performed according to an established local standard (for further details, see Østergaard et al. $\left.^{36}\right)$. All PD DBS candidates at Aarhus University Hospital undergo preoperative MRI scans under general anesthesia for optimal surgical planning data. For the enrolled patients with PD, this scanning session was extended to allow acquisition of high-resolution DWI data. To further examine the robustness of the diffusion acquisition and tractography results, we included 1 healthy adult (31 years old), who underwent repeated MRI sessions over a 4-week period. All participants gave written informed consent and the study was approved by the local ethics committee.

\section{Image Acquisition}

Two weeks before surgery, each patient with PD underwent the extended preoperative MRI session (2 hours) to acquire standard T1- and T2-weighted MRI for DBS planning, along with high-resolution DWI for tractography. The healthy participant was scanned 10 times with an identical DWI protocol over a 4-week period (nonanesthetized, but with minimal movement).

All MRI data were acquired on a 3-T Siemens Tim Trio using a 32-channel head coil. A 3D T1-weighted magnetization-prepared rapid-acquisition gradient echo sequence was obtained with the following parameters: TR 2420 msec; TE 4.58 msec; matrix $256 \times 256$; slices 176 (sagittal); slice thickness $1 \mathrm{~mm}$; resolution $1 \mathrm{~mm}$ isotropic; and averages 2 .
A T2-weighted turbo spin echo sequence was acquired with the following parameters: TR $3470 \mathrm{msec}$; TE 77 msec; matrix $384 \times 384$; slices 30 (axial); slice thickness $1.5 \mathrm{~mm}$; and resolution $0.625 \times 0.625 \times 1.5 \mathrm{~mm}$.

A high-resolution DWI sequence was acquired using a readout segmentation of long variable echo trains (RESOLVE) $)^{40}$ sequence. In addition to the reduction in geometrical distortions (Poster presentation by Petersen MV et al., Organization for Human Brain Mapping Annual Conference, 2014) provided by segmented readout, the RESOLVE sequence uses navigator echoes and realtime reacquisition to reduce the effects of cardiac pulsation, which are pronounced in the brain below the level of the corpus callosum. ${ }^{41}$ We used the following parameters: 62 diffusion-encoding directions, with a b value of $1000 \mathrm{sec} / \mathrm{mm}^{2}, 9$ interleaved nondiffusion-weighted $(\mathrm{b}=$ 0) volumes; TR 13,500 msec; TE $81 \mathrm{msec}$; matrix $136 \times$ 136; slices 84 (axial); slice thickness $1.4 \mathrm{~mm}$; resolution $1.4 \mathrm{~mm}$ isotropic; readout segments 5, leading to an acquisition time of approximately 1 hour and 30 minutes. To facilitate further reduction of susceptibility-induced distortions, we acquired a set of $4 \mathrm{~b}=0$ images with similar parameters but with opposing phase-encoding polarity.

\section{Data Processing of MRI Studies}

Figure 1 presents the full processing pipeline, which used the following toolboxes: the FreeSurfer image analysis suite (http://surfer.nmr.mgh.harvard.edu), ${ }^{10}$ ITK-SNAP (http://www.itksnap.org), ${ }^{52}$ FSL (FMRIB Software Library v.5.0, Oxford Centre of Functional MRI of the 


\section{A $\mid$ manual STN segmentation (T2)} B | automatic motor cortex parcellation (T1)
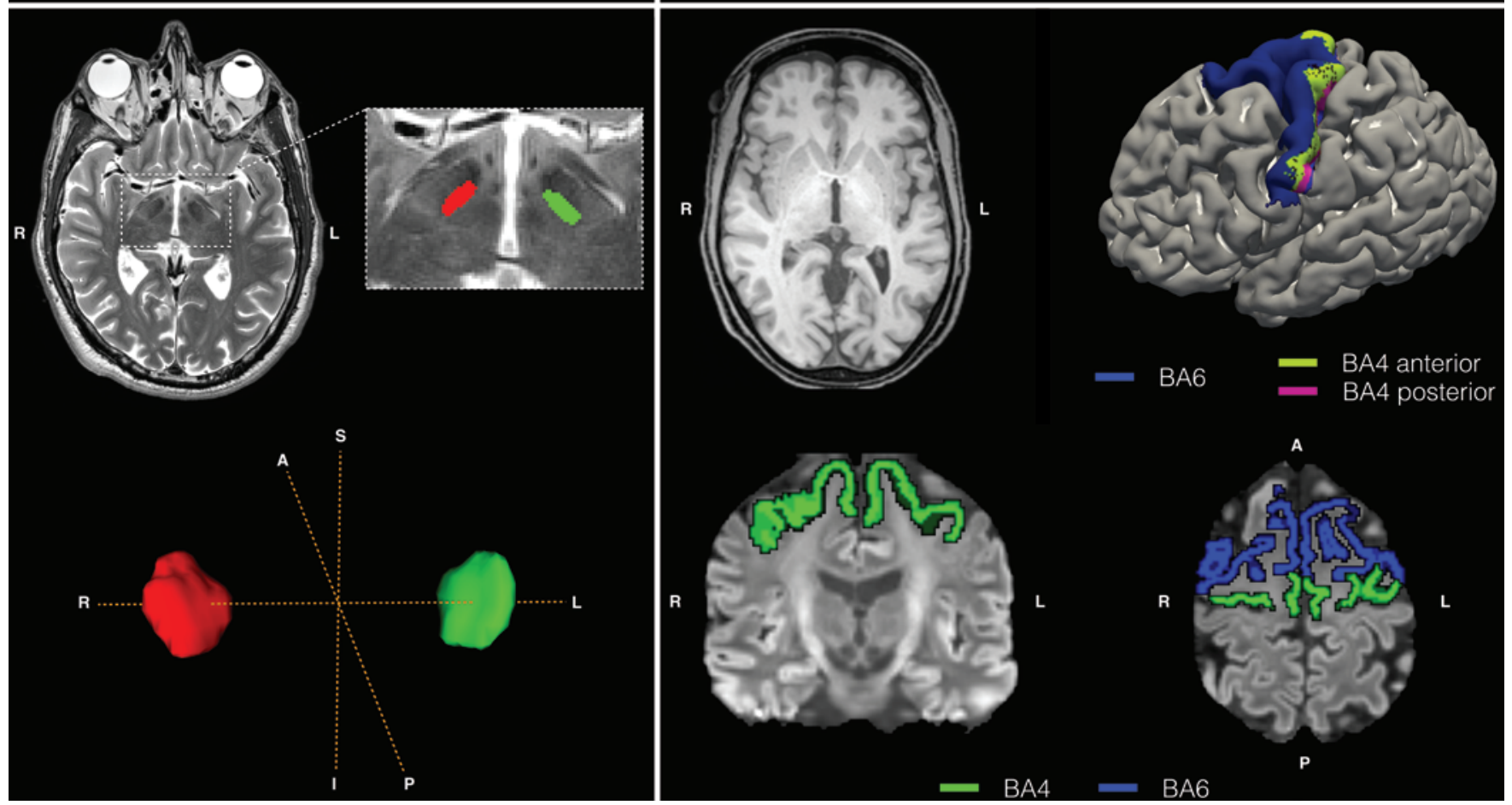

FIG. 2. A: The STN is manually segmented using the T2-weighted scan (ITK-SNAP). B: The T1-weighted scan is used to generate a patient-specific parcellation of the cortical motor regions defined by BA4 and BA6 (FreeSurfer). $A=$ anterior; $I=$ inferior; $L=$ left; $P=$ posterior; $R$ = right; $S$ = superior. Figure is available in color online only.

Brain, http://fsl.fmrib.ox.ac.uk), ${ }^{18}$ and the MRtrix3 software package (https://github.com/jdtournier/mrtrix3). ${ }^{43}$

\section{Region-of-Interest Segmentation}

Figure 2 presents the segmentation procedure. For accurate delineation of the STN and generation of individualized processing masks, a functional neurosurgeon with $>20$ years of clinical experience (N.S.) manually segmented the nuclei on the T2-weighted scan using ITKSNAP.

FreeSurfer was used to analyze the T1-weighted scan and to automatically parcellate cortical regions based on individual patient anatomy. Probabilistic maps of the cortical regions corresponding to BAs 4 and 6 were extracted and combined to create a motor cortex mask. The generated masks (STN and BA4 + BA6) were transformed to the coordinate system of the diffusion data using a 6-degreesof-freedom rigid-body registration between MRI modalities (FSL).

\section{Diffusion Data Preprocessing}

The diffusion data were preprocessed using FSL to correct for DWI-related artifacts, including correction of motion, eddy currents, EPI distortions (caused by B0 field inhomogeneity), ${ }^{2}$ and intensity inhomogeneity (bias field). Next, the MRtrix toolbox was used to analyze the raw diffusion signal and generate fiber orientation estimates, using 1) the DT model and 2) a higher-order CSD-based diffusion model. Total processing time on a $3.5-\mathrm{GHz} 6$-core
Intel Xeon E5-equipped workstation was approximately 4 hours.

\section{Tractography Framework}

Figure 3 illustrates relevant steps of the tractography analysis. We performed an identical tractography analysis using 2 different techniques, corresponding to the 2 fitted diffusion models: a deterministic (DT model) and probabilistic (CSD model) algorithm as implemented in the MRtrix toolbox. For simplicity, we refer to the deterministic method as DT and the probabilistic method as CSD throughout. Default tracking parameters were used for both algorithms except for amplitude cutoff values, which for both were increased to 0.2 (default, 0.1) to limit the number of erroneous streamlines generated (see Fig. 4 for detailed tracking parameters).

The following steps explain the details of our tractography procedure (see Fig. 3). First, 100,000 streamlines were initiated randomly from a seed region covering the motor cortex (BAs $4+6)$. The streamlines were not constrained by any target, inclusion, or exclusion regions and would only terminate when exceeding the predefined cutoff values. Second, using the manually generated STN segmentation, we extracted the streamlines that terminated within the STN region, corresponding with a direct cortico-subthalamic connection. Third, using a simple streamline counting approach, we generated a track-density image (TDI) of the delineated left and right corticosubthalamic streamlines. Briefly, the number of stream- 


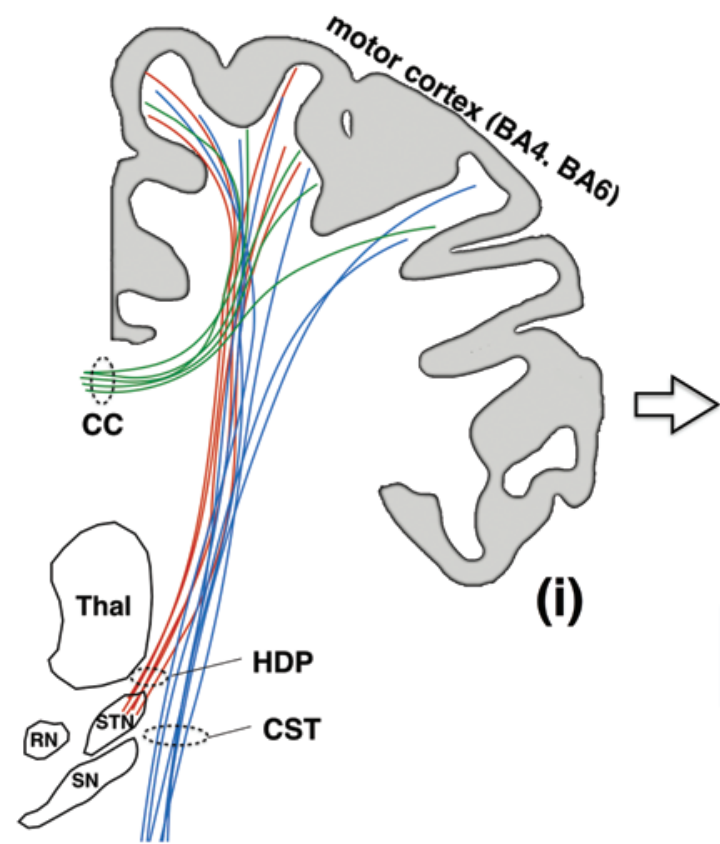

\section{A | schematic illustration}

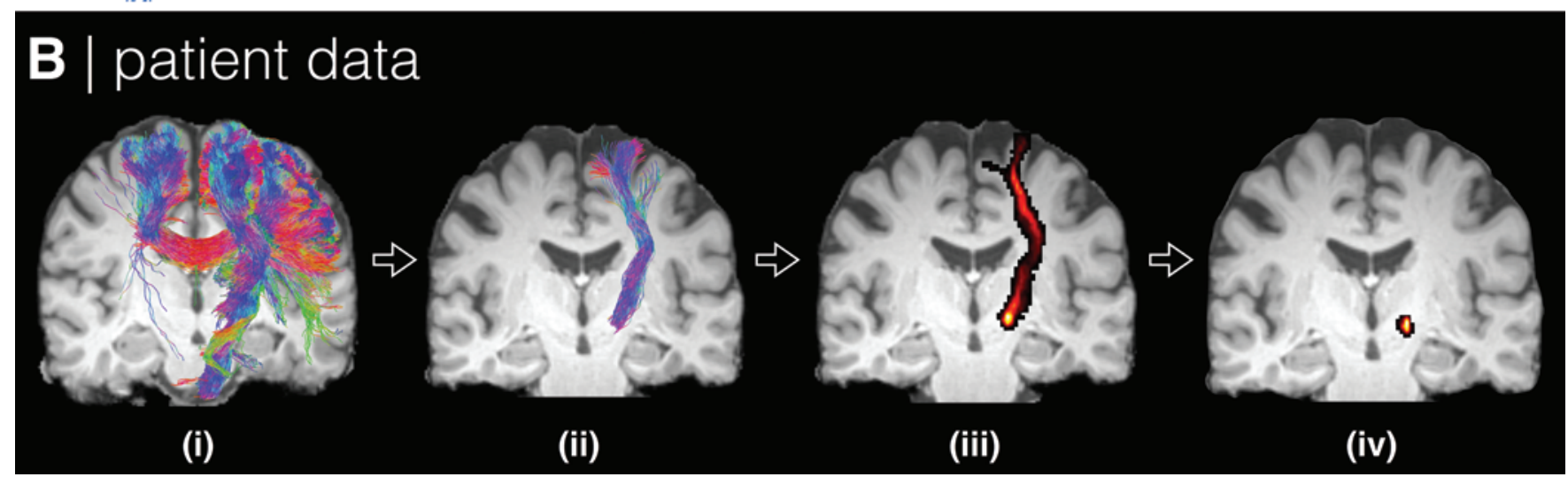

FIG. 3. A: Schematic illustration of the tractography analysis: HDP delineation and STN target map creation. Raw tractography results consisting of 100,000 streamlines seeded throughout the motor cortex in 1 hemisphere (i). Unfiltered results are dominated by streamlines following the CST and crossing through the corpus callosum. Streamlines that terminate within the boundaries of the STN mask are extracted, representing the HDP (ii). The HDP streamlines are resampled into a TDI. See Methods for further details (iii). The TDI is masked using the STN mask, resulting in a probability map that identifies the region within the STN with a high density of HDP streamlines (iv). This final map thus represents a tractography-derived HDP-STN target map. B: Results from a patient data set corresponding to the steps outlined above (CSD method illustrated). CC = corpus callosum; RN = red nucleus; $\mathrm{SN}=$ substantia nigra; Thal = thalamus. Figure is available in color online only.

lines passing through a given intersected voxel was counted and mapped as a numerical value for this voxel. Fourth, using the STN segmentations again, we masked the TDI to generate an STN map depicting the areas within the STN that demonstrated a high density of streamlines connecting to the cortical motor areas. Fifth, by merging these voxel-based TDI target maps with the coregistered (intensity-scaled) T1-weighted image, we generated a new fusion T1-weighted HDP image that can be readily exported (DICOM format) and loaded in standardized neuronavigation systems.

This processing pipeline results in 2 STN maps representing HDP target regions, 1 using the DT-based and 1 using the CSD-based tractography method. To contrast the 2 STN target regions identified in the patients with
$\mathrm{PD}$, we extracted coordinates for the center of gravity and calculated the distance between the 2 points.

To compare the target robustness across the 10 repeated scans, we again extracted the center of gravity and calculated the standard deviation of the 2 methods across the $x-y-z$ axes. To quantify the variability of the 2 methods, we combined the standard deviations across axes into a vector and calculated the length.

\section{Results}

\section{Delineation of the HDP}

Figure 4 presents the raw cortico-subthalamic tracking results using our analysis framework. Both the DT and CSD methods successfully reconstructed a pathway 


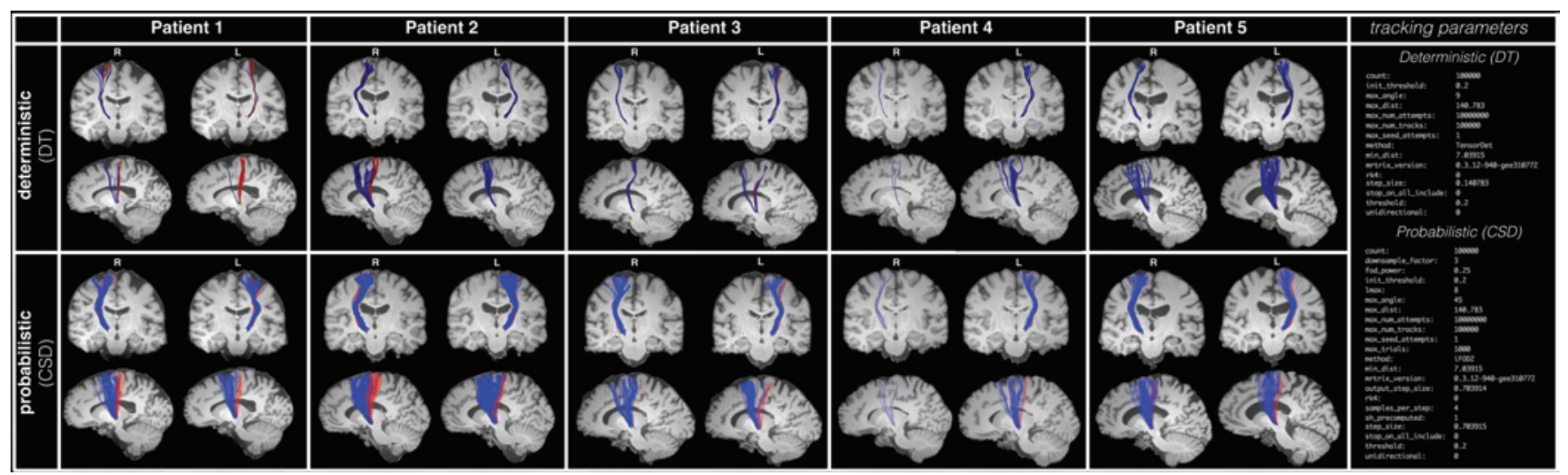

FIG. 4. Tractography results of the delineated hyperdirect cortico-subthalamic streamlines across all 10 hemispheres. Red denotes streamlines connecting the STN and BA4; blue streamlines connect with BA6. The upper row shows the DT-based deterministic method, and the lower row shows the CSD-based probabilistic technique. Where the red and blue streamlines appear to follow the same path, they are connecting to separate cortical regions near the boundary between the FreeSurfer BA4 and BA6 parcellations. See Results for further description. Figure is available in color online only.

between the motor cortex and ipsilateral STN in all 5 patients and across all 10 STNs. The DT method reconstructed narrow and sparse bundles of streamlines originating from small and highly variable areas of the motor cortices. In 6 of 10 patient hemispheres, this method delineated connections only from the BA6 region. In contrast, the CSD method overall reconstructed a more continuous set of streamlines projecting to the STN from a larger area of the segmented cortical motor regions. The CSD method identified a larger proportion of streamlines connecting BA6 and the STN, compared with connections from BA4 that appeared to be fewer and more variable across patients. All reconstructions across both methods appeared to connect the motor cortices with the dorsolateral aspect of the ipsilateral STN and to course through a segment of the internal capsule en route to the STN. In the right hemisphere of the patient in Case 4, both methods identified only a limited number of streamlines.

\section{The STN-HDP Target Region: Patients With PD}

With both tractography methods, we identified HDP target regions that lie within the dorsolateral aspect of the STN. There is an overlap between the 2 defined target regions in all subthalamic nuclei. This overlap varies considerably, both between patients and between hemispheres in individual patients. The difference is highlighted when examining the peak intensity region (representing the highest density of HDP streamlines) of the STN target maps (Fig. 5). Generally, it appears that the CSD method identified a larger and more diffuse region of the STN compared with the DT method. Comparing center-of-gravity coordinates yielded by the 2 methods resulted in a difference of $0.5-2.6 \mathrm{~mm}$ (mean $1.41 \mathrm{~mm}, \mathrm{SD} 0.62$ ). In fact, across these 2 methods, half of the center coordinates differed by $>1.5 \mathrm{~mm}$ (summarized in Table 1).

\section{Robustness of Tractography Results: Healthy Control Participant}

Figure 6A and B illustrates the consistency of the target maps generated across the 10 repeated scans obtained in the healthy participant using the 2 methods. The overall target area and peak streamline density appear more variable in the DT method. In contrast, as demonstrated in Figure 6B, the CSD target maps are more consistently confined to the posterolateral aspect of the STN outline. When we compared the center-of-gravity $x-y-z$ coordinates across the 10 repeated scans, we found that the DT method is considerably more variable than the CSD method (Table 2). To calculate a spatial metric for this variation, we combined the standard deviations across the $\mathrm{x}-\mathrm{y}-\mathrm{z}$ axes and found that the DT method demonstrated an inaccuracy of $0.8-1.3 \mathrm{~mm}$, which was 1.9-2.4 times higher than for the CSD-derived targets (illustrated in Fig. 6C).

\section{Exportation to Neuronavigation Software}

We can interpret the delineated STN target regions as probability maps representing the likelihood of HDP connectivity (Fig. 7). The generated T1-weighted HDP image combines the tractography-derived target maps with the anatomical information necessary to coregister and fuse it with traditional stereotactic DBS planning data (CT, T1-, and T2-weighted scans). Figure 8 demonstrates the T1-weighted HDP target map imported and merged with a patient's preoperative stereotactic targeting plan in the SurgiPlan neuronavigation software.

\section{Discussion}

In this study, we have delineated the cortico-subthalamic HDP by using high-resolution DWI data and 2 commonly used tractography techniques: 1) a simple DT-based deterministic method and 2) a more advanced CSD-based probabilistic method. We examined the application of these 2 methods in a sample of patients with PD who were selected for DBS treatment, where stimulation of specific white matter pathways is crucially linked to treatment outcomes. Across all patients, the DT-based method reconstructed a smaller and more variable subset of streamlines between the STN and the 2 defined cortical motor areas BA4 and BA6, compared with the CSD method. This vari- 


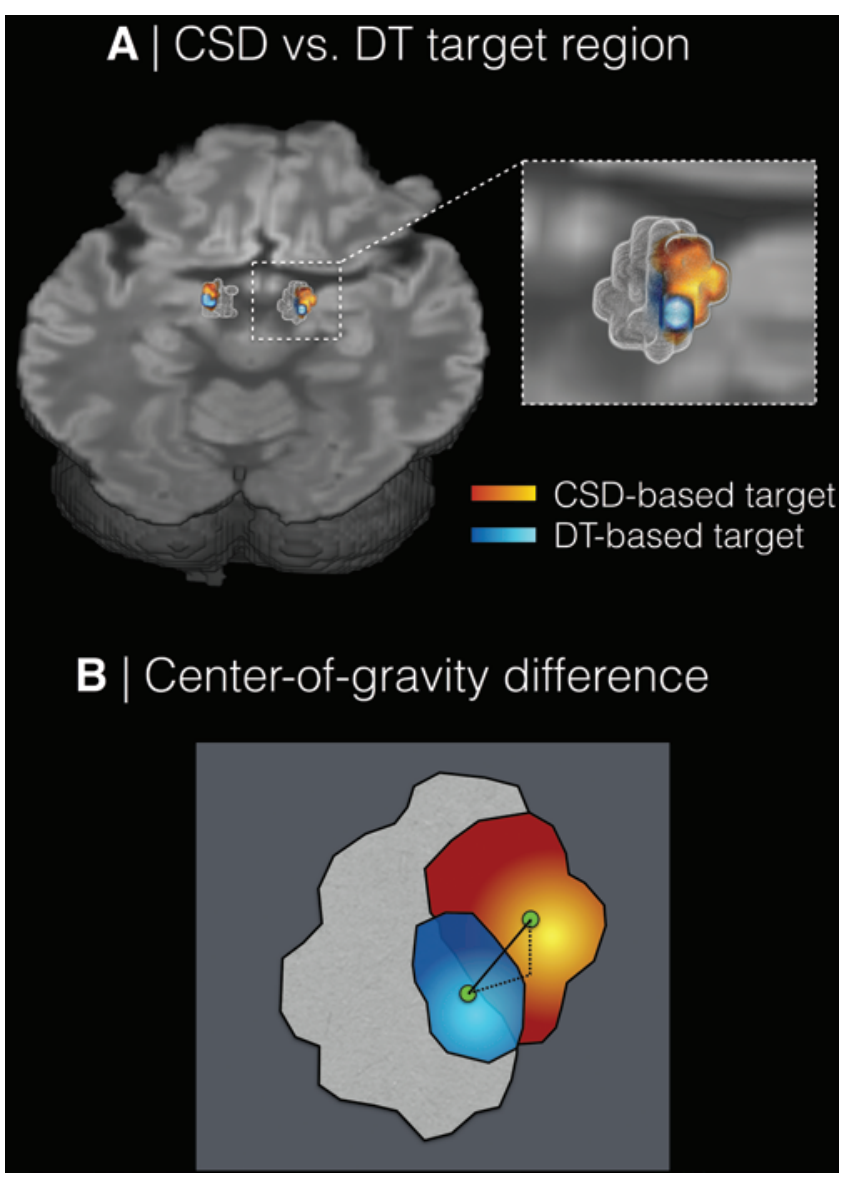

FIG. 5. A: A 3D view (axial slice) outlining the entire STN (white) and the target regions defined using probabilistic (yellow-red) and deterministic (white-blue) tractography methods. B: Schematic illustration of the DTand CSD-derived target region's center of gravity and calculation of the difference between the 2 methods. Figure is available in color online only.

ability is easily appreciated when examining the resulting streamlines in the sagittal slices, shown in Figure 4. The CSD method delineates a continuous set of streamlines connecting a more dispersed area of motor cortex to the STN. This is important because a reliable, consistent delineation of this pathway is necessary if tractography methods are to be applied to the neurosurgical treatment planning of PD.

In 1 of the patients, the right hemisphere cortico-subthalamic connections appeared to be particularly diminished, as reconstructed by both the deterministic and probabilistic methods. Clinically, this patient was different by virtue of diagnosis of dopa-responsive dystonia at an early age confirmed by genetic testing, followed by a diagnosis of idiopathic PD confirmed by a dopamine transporterSPECT scan. It is plausible that this individual had greater neural degeneration compared with the other 4 patients. Several recent tractography-based studies have identified structural white matter abnormalities and disruption of fiber pathways in cerebello-thalamo-cortical networks of patients with idiopathic dystonia. ${ }^{5,47}$ In the present patient, the clinical PD presentation was dominated by leftsided symptoms consistent with right-sided degeneration, as modeled here. Further work on movement disorder in patients with varying stages of disease might enable us to disentangle disorder-related neural degeneration from potential technical limitations of these methods.

To assess and quantify the divergence between the 2 methods, we compared the center of gravity of the tractography-derived target maps generated. We found a substantial difference between methods, with an average difference of $>1.4 \mathrm{~mm}$ and half of the targets differing by $\geq 1.5 \mathrm{~mm}$ (Table 1). This difference is of a significant magnitude when compared with the accuracy of current stereotactic DBS electrode-placement procedures. ${ }^{7,13}$ Furthermore, when assessing the consistency of generated STN targets across 10 repeated scans obtained in a single healthy adult, we found that the DT method produced substantially more variable results (Fig. 6). Across all scans, the CSD technique delineated a more consistent target region located toward the dorsolateral region of the STN. The robustness of the probabilistic method was further highlighted when comparing the center-of-gravity coordinates across the 10 scans, where the DT method demonstrated a highly variable pattern by comparison (Table 2). This further signifies that the tensorbased method is insufficient for many clinical aims.

Although it was only possible to obtain repeated measurements in the healthy participant, it is unlikely that the variation observed between the 2 methods (Table 2) would be less pronounced in a patient sample. A large proportion of the variation seen across patients (Table 1) can probably be attributed to methodological insufficiencies related to the DT method ( $0.8 \mathrm{~mm}$ left, $1.3 \mathrm{~mm}$ right). Furthermore, the data acquired here were of very high quality compared with typical clinical acquisitions. We would not expect the DT method to provide more reliable results with lowerquality data.

The technical inadequacy of DT-based tractography is established in nonclinical neuroimaging studies. ${ }^{19-23}$ In patients undergoing resection of tumors in close proximity to eloquent cortical areas, it has been demonstrated that deterministic algorithms tend to grossly underestimate the extent of the corticospinal tract (CST). Typically, only a narrow subset of streamlines projecting from the medial

TABLE 1. Difference in STN target region center-of-gravity coordinates between DT-based and CSD-based tractography methods

\begin{tabular}{ccccc}
\hline \multirow{2}{*}{$\begin{array}{c}\text { Case } \\
\text { No. }\end{array}$} & Hemisphere & \multicolumn{2}{c}{ Center-of-Gravity Coordinates } & Distance, \\
\cline { 2 - 5 } & DT & CSD & mm \\
\hline \multirow{2}{*}{1} & Lt & $-9.4,17.0,3.5$ & $-9.1,18.4,3.8$ & 1.5 \\
\cline { 2 - 5 } & Rt & $15.4,16.9,1.9$ & $16.0,18.6,2.6$ & 1.9 \\
\hline 2 & Lt & $-10.1,6.5,-6.6$ & $-11.3,5.9,-6.8$ & 1.4 \\
\cline { 2 - 5 } & Rt & $12.2,8.5,-9.6$ & $12.9,8.1,-10.1$ & 0.9 \\
\hline \multirow{2}{*}{3} & Lt & $-17.7,8.8,4.6$ & $-17.9,9.2,6.0$ & 1.5 \\
\cline { 2 - 5 } & Rt & $7.9,6.1,4.8$ & $7.8,7.0,6.3$ & 1.8 \\
\hline \multirow{2}{*}{4} & Lt & $-18.3,11.8,5.5$ & $-18.7,12.0,5.7$ & 0.5 \\
\cline { 2 - 5 } & Rt & $5.05,11.4,6.3$ & $5.02,13.6,5.1$ & 2.6 \\
\hline 5 & Lt & $-11.5,21.2,3.5$ & $-11.5,21.5,2.9$ & 0.7 \\
\cline { 2 - 5 } & Rt & $14.4,22.7,1.5$ & $15.4,21.9,1.5$ & 1.3 \\
\hline & & & & Mean 1.41 \\
\hline
\end{tabular}




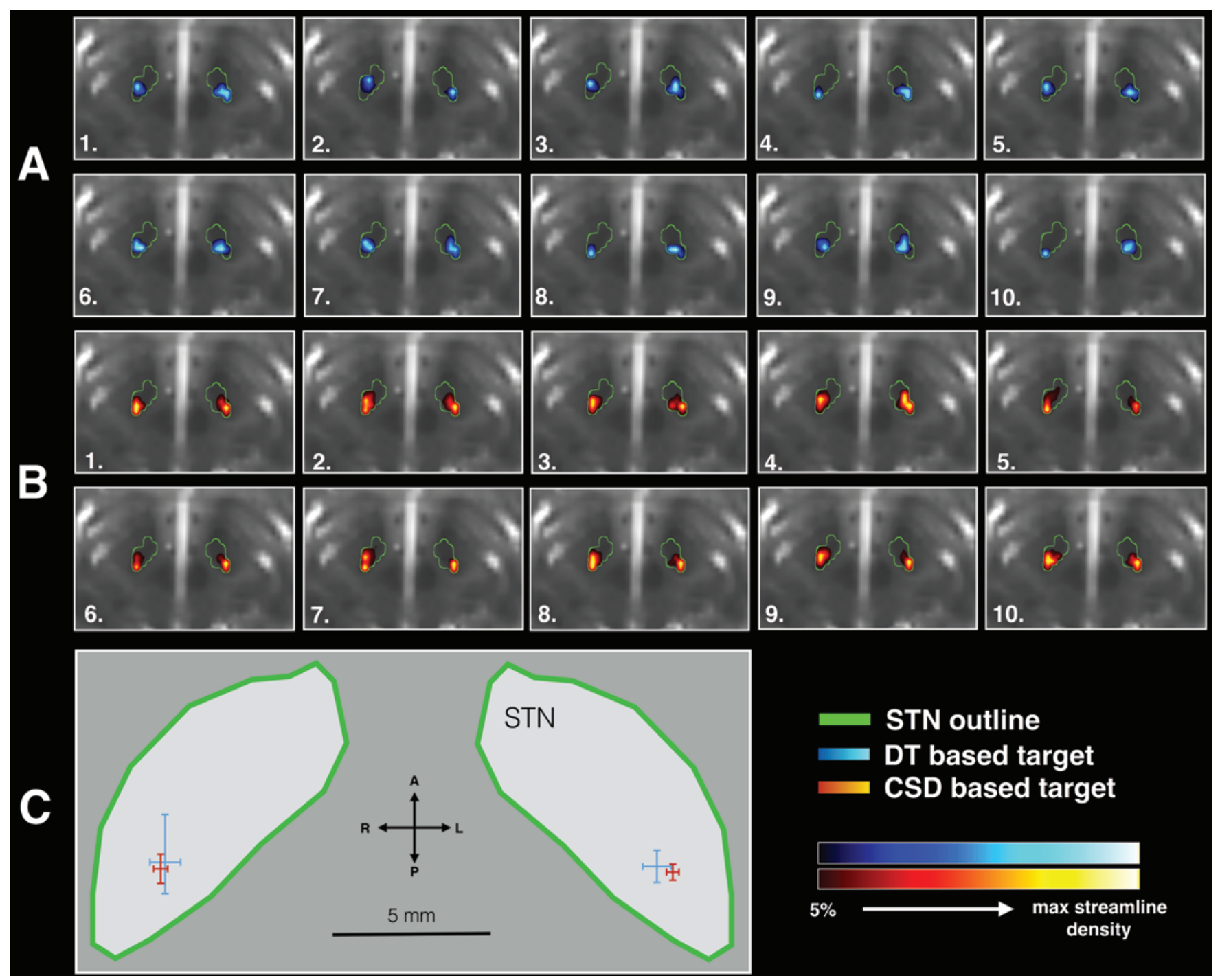

FIG. 6. A: Axial plane (through the dorsal third of the STN) depicting the DT-based target maps generated across 10 repeated scans obtained in a healthy control participant. B: Axial plane at the same level depicting the CSD-based targets across repeated scans. C: Schematic figure of the STN illustrating the standard deviation of the calculated center-of-gravity coordinates along the anterior-posterior and right-left axes. Figure is available in color online only.

aspects of the motor cortex are identified, ${ }^{11}$ similar to the findings reported here for the HDP. The improved anatomical accuracy of probabilistic compared with deterministic tractography has furthermore been demonstrated in these patients by using intraoperative electrical stimulation of subcortical white matter and cortical motor neurons. ${ }^{30}$ The CSD-based method implemented in the current work has demonstrated robust results on both phantom and clinical data. ${ }^{25,45,50}$ Although the model is especially well suited to diffusion data acquired with a high b value $(>2000 \mathrm{sec} /$ $\mathrm{mm}^{2}$ ), where it can resolve crossing fibers at an angle as low as $35^{\circ}$, it has also shown robust results on data acquired at typical clinical b values $\left(1000-1200 \mathrm{sec} / \mathrm{mm}^{2}\right)$, provided that a high number of directions are acquired..$^{50}$

The small number of studies using probabilistic tractography to examine STN connectivity in healthy participants all report some degree of connectivity between cortical motor areas and the STN, suggesting that the HDP can be identified in vivo in humans. Only 2 of these studies provide specific details on the anatomical course of the connections between the STN and cortical motor areas, ${ }^{6,51}$ whereas other studies simply note the presence of these connections. ${ }^{1,26}$ Furthermore, 1 of these 2 studies reported the identification of this pathway only in a minority of participants, suggesting inconsistency in methodological output. ${ }^{6}$ The current probabilistic method plausibly delineated the HDP across all of the patients tested. Furthermore, the technique we have implemented reconstructed STN connectivity with both BA4 and BA6. This is consistent with recent primate studies using anterograde neuronal tracing and reporting an HDP from both BA4 and BA6. ${ }^{16}$

The current delineation of the HDP is consistent with a recent tensor-based tractography study of patients treated with DBS. ${ }^{4}$ In this study, the authors reported that they could define an STN target region closer to the final (optimized through test stimulation) electrode position than to the origi- 
TABLE 2. Variability of STN target region center-of-gravity coordinates between DT-based and CSD-based tractography techniques across 10 repeated scans

\begin{tabular}{ccccc}
\hline & \multicolumn{2}{c}{$\begin{array}{c}\text { Standard Deviation of Center-of- } \\
\text { Gravity Coordinates }\end{array}$} \\
\cline { 2 - 3 } $\begin{array}{c}\text { Tractography } \\
\text { Method }\end{array}$ & $\begin{array}{c}\text { Medial- } \\
\text { Lateral }\end{array}$ & $\begin{array}{c}\text { Anterior- } \\
\text { Posterior }\end{array}$ & $\begin{array}{c}\text { Superior- } \\
\text { Inferior }\end{array}$ & $\begin{array}{c}\text { Length, } \\
\mathrm{mm}\end{array}$ \\
\hline Lt hemisphere & & & & \\
\hline DT & 0.49 & 0.43 & 0.48 & 0.81 \\
\hline CSD & 0.20 & 0.26 & 0.27 & 0.43 \\
\hline Ratio & & & & 1.90 \\
\hline Rt hemisphere & & & & \\
\hline DT & 0.47 & 1.15 & 0.51 & 1.34 \\
\hline CSD & 0.23 & 0.44 & 0.25 & 0.56 \\
\hline Ratio & & & & 2.42 \\
\hline
\end{tabular}

nal planned target. This is encouraging, given the relatively low nominal resolution of their data (2.6-mm isotropic voxels) and deterministic framework; it provides impetus for explorations with higher-resolution acquisitions and probabilistic tractography, such as implemented in the current study. In general, our results support these earlier indications.

Our current work has implemented a readout-segmented EPI diffusion acquisition, with substantially higher nominal resolution than previous studies (1.4-mm isotropic voxels). In contrast to single-shot EPI, this acquisition resulted in an actual resolution only slightly worse than the nominal. This higher resolution provided us with $>6$ times as many voxels covering the STN, allowing us to characterize regions of connectivity within the STN in far greater detail. With these data, we have demonstrated that choice of tractography method has a significant impact on the delineation of a target region within the STN. The larger, diffuse target region defined by probabilistic tractography is in part due to the technical nature of the method, incorporating uncertainty of the estimated fiber orientations. Consequently, we have chosen to compare the center of gravity of the generated target maps.

Last, we have described a framework for generating an STN target map by converting the tractography-derived streamlines into a voxel-based TDI. This is of significant interest to clinicians because such a target map can be converted to the standardized DICOM format and imported into surgical neuronavigation systems. Clinical implementation of advanced tractography methods has hitherto not been explored because of constraints within these surgical planning systems. The TDI format supports easy conversion and import because it is a simple voxel-based intensity map as opposed to the raw 3D streamlines that are generated with tractography.

Despite the widely discussed limitations of the DT model and deterministic tractography technique, it is still being used in clinical neurosurgical studies. ${ }^{4,11}$ This may be due, in part, to the constraints of commercially available neuronavigation systems. None of these currently support probabilistic tractography, and only a few have implemented basic deterministic methods. In this study, we have exported the MRI data to a custom workstation

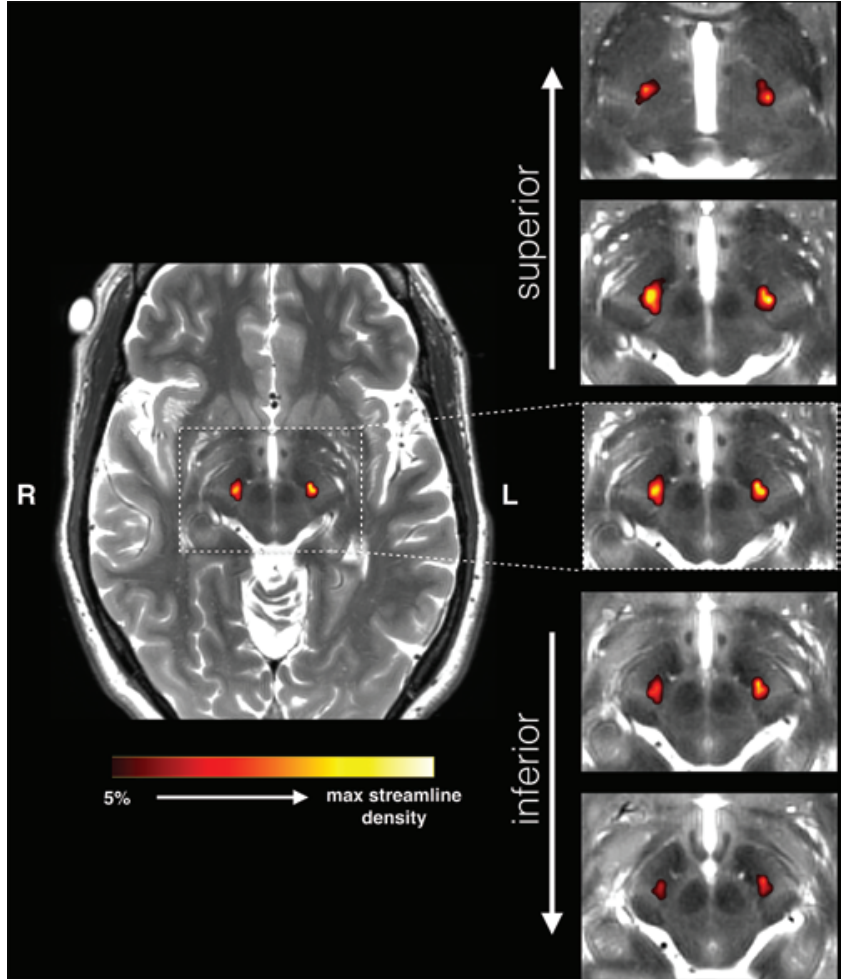

FIG. 7. Axial slices (anterior commissure-posterior commissure aligned) depicting the HDP-STN target map (CSD-based) overlaid on the T2weighted image traditionally used for targeting. Figure is available in color online only.

set up for data processing. This allows us to implement more advanced preprocessing and tractography methods than commercially available. Diffusion acquisitions are very sensitive to patient movement and are affected by several characteristic artifacts, leading to geometrical distortions (for further details, see Jones ${ }^{21}$ and Tournier et al. $\left.{ }^{44}\right)$. Corrections of these distortions are essential for analysis of the raw diffusion signal and are furthermore crucial for achieving accurate coregistration with undistorted (T1- and T2-weighted) MRI modalities. The current study, and the majority of other tractography studies, relies on segmentation of structures on anatomical scans, which are then transformed and assumed to line up correctly on diffusion scans. The optimal distortion correction requires tools that are not currently available in any neuronavigation system, as well as the additional acquisition of diffusion data with reverse phase encoding, which has so far limited the implementation in clinical studies.

In our proposed framework, the only requirement for manual input is for segmentation of the STN. We aimed for the most accurate segmentation possible, to evaluate the impact of 2 divergent tractography methods. However, another methodological advantage of our study is that we initiated streamlines from the motor cortex and extracted only streamlines that terminated in the defined STN region. If the STN region was overestimated and included parts of the nearby CST, we would still only extract HDP streamlines as those that terminated within the region. This would effectively filter out corticospinal streamlines that pass through the region. In future work, this process 


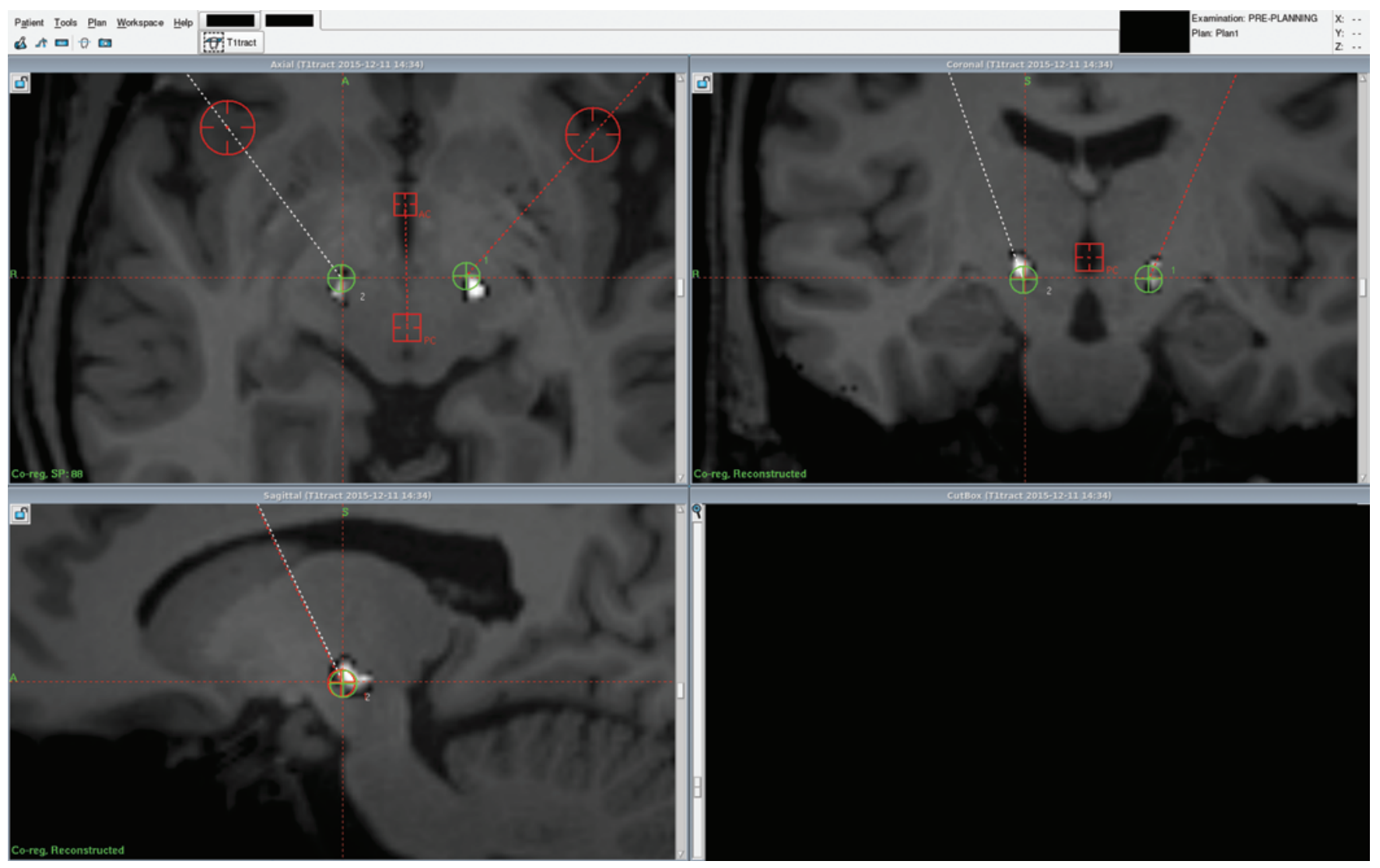

FIG. 8. Example of the fusion T1-weighted HDP targeting map imported and registered with preoperative planning data in neuronavigation software (Leksell SurgiPlan, Elekta). The screenshot shows an axial, coronal, and sagittal slice through the planned STN targets (sagittal view is focused on the right STN). The green target reticle depicts the originally planned target. The bright white area is the probability map of the tractography-derived STN target (CSD) and in this patient demonstrates proximity to the traditional target. Note that tractography data were not made available to neurosurgeons before DBS surgery. Therefore, the preoperatively planned DBS targets were not adjusted based on the tractography-derived targets, and the T1-weighted HDP target map was overlaid to illustrate the feasibility. Figure is available in color online only.

could be fully automated by using available probabilistic atlas models of the $\mathrm{STN}^{24}$ and thus could provide targeting maps for the surgeon performing DBS, with no need for manual inputs.

The major limitation of the current study is the timeconsuming readout-segmented echo-planar DWI acquisition used (i.e., RESOLVE). This acquisition allowed us to acquire high-resolution DWI data with a great reduction of geometrical distortions and other artifacts (cardiac pulsation and motion), but at the cost of long acquisition time. However, with ongoing improvements in MR scanner hardware ${ }^{46}$ and acquisition protocols, we can expect such lengthy scan times to be reduced by a factor of $\geq 3$ in the near future. ${ }^{14}$ In addition, reducing the number of unique diffusion-encoding directions, or limiting the field of view to only a set of coronal slices covering the STN and cortical motor areas, could potentially shorten time requirements further.

A further constraint in our protocol is that patients were anesthetized, which may not be feasible in all clinical settings. For the current study, this allowed us to obtain optimal-quality data. Furthermore, it is challenging to avoid movement artifacts in nonanesthetized patients or healthy participants in prolonged scanning sessions. Finally, the field of tractography more generally is faced with challenges such as the lack of anatomical validation and the lack of consensus on analytical procedures.

The unconstrained framework of this tractography study is not designed to assess the integrity of prespecified pathways, but rather to explore if relevant pathways can be plausibly delineated and targeted using in vivo tractography methods. Further refinement is necessary if we are to systematically compare and quantify the integrity of the discussed pathways across patients. This includes adjusting for the size of parcellated motor and STN regions, as well as tracking both from the cortex to the STN and from the STN to the cortex. Furthermore, the cortical motor regions could be manually segmented into the premotor, supplementary, and primary motor cortex. This could allow us to further quantify connectivity with the specific cortical motor regions. It would also be of interest to examine if STN regions dominated by connectivity with either of the motor regions can be identified, as has been demonstrated in a recent primate tracing study. ${ }^{16}$ Finally, the probabilistic method identified a varying number of streamlines from the primary motor cortex (BA4) across all patients. However, it is difficult to evaluate how much of this variation is related to actual structural differences 
between patients, or to variation in the FreeSurfer parcellation, which uses probabilistic maps to approximate BAs.

Together with innovations such as directional stimulation, better targeting provides a clear means to improve treatment outcome. ${ }^{29,39}$ Our tractography framework demonstrates an avenue for refining and individualizing DBS treatment planning. Future studies will be able to extend the current approach to delineating limbic STN projections. This could be helpful in neurosurgical DBS planning for either PD, where we aim to avoid this region, or for obsessive-compulsive disorder, where we aim to target this region. ${ }^{9}$ Further studies using larger patient cohorts are required to validate improved treatment efficacy of tractography-based targeting over traditional STN-targeting methods. The gold standard methodology would ultimately be randomized controlled trials. However, retrospective studies-combining tractography with data on electrode position, stimulation fields, and clinical outcomes-would allow us to investigate the structural networks modulated with DBS. This would potentially allow characterization of the STN connectivity patterns associated with positive and negative treatment effects.

\section{Conclusions}

Our study clearly demonstrates that the CSD-based probabilistic method provides considerably more consistent and plausible tractography results compared with the DT-based method. If tractography is to be implemented in future neurosurgical treatment planning, it is crucial that we use a robust and reliable method, such as the CSDbased framework presented here. Although DT-based tractography is available in some commercial neuronavigation software, its demonstrated limitations render it inadequate for clinical applications, where accurate stereotactic targeting is required.

\section{Acknowledgments}

We gratefully acknowledge the technical assistance provided by Dora Zeidler and Ryan Sangill. Mikkel V. Petersen was supported by a generous private donation from K. Klastrup. Torben E. Lund was supported by the Danish Ministry of Science, Technology and Innovation's UNIK program (MINDLab). Karen Østergaard was supported by the Lundbeck Foundation, the Danish Council for Independent Research, the Danish Parkinson Association, the Central Denmark Research Foundation, Aarhus University, and Aarhus University Hospital. The scanner was funded by a grant from the Danish Agency for Science, Technology and Innovation. Thanks to Siemens Healthineers, for providing access to the RESOLVE sequence (WIP544A) for the Trio scanner.

\section{References}

1. Accolla EA, Dukart J, Helms G, Weiskopf N, Kherif F, Lutti A, et al: Brain tissue properties differentiate between motor and limbic basal ganglia circuits. Hum Brain Mapp 35:5083-5092, 2014

2. Andersson JL, Skare S, Ashburner J: How to correct susceptibility distortions in spin-echo echo-planar images: application to diffusion tensor imaging. Neuroimage 20:870-888, 2003

3. Assaf Y, Pasternak O: Diffusion tensor imaging (DTI)-based white matter mapping in brain research: a review. J Mol Neurosci 34:51-61, 2008

4. Avecillas-Chasin JM, Alonso-Frech F, Parras O, Del Prado N, Barcia JA: Assessment of a method to determine deep brain stimulation targets using deterministic tractography in a navigation system. Neurosurg Rev 38:739-751, 2015

5. Bonilha L, de Vries PM, Hurd MW, Rorden C, Morgan PS, Besenski N, et al: Disrupted thalamic prefrontal pathways in patients with idiopathic dystonia. Parkinsonism Relat Disord 15:64-67, 2009

6. Brunenberg EJ, Moeskops P, Backes WH, Pollo C, Cammoun L, Vilanova A, et al: Structural and resting state functional connectivity of the subthalamic nucleus: identification of motor STN parts and the hyperdirect pathway. PLoS One 7:e39061, 2012

7. Burchiel KJ, McCartney S, Lee A, Raslan AM: Accuracy of deep brain stimulation electrode placement using intraoperative computed tomography without microelectrode recording. J Neurosurg 119:301-306, 2013

8. Castrioto A, Lhommée E, Moro E, Krack P: Mood and behavioural effects of subthalamic stimulation in Parkinson's disease. Lancet Neurol 13:287-305, 2014

9. Chabardes S, Polosan M, Krack P, Bastin J, Krainik A, David $\mathrm{O}$, et al: Deep brain stimulation for obsessive-compulsive disorder: subthalamic nucleus target. World Neurosurg 80:S31. e1-S31.e8, 2013

10. Destrieux C, Fischl B, Dale A, Halgren E: Automatic parcellation of human cortical gyri and sulci using standard anatomical nomenclature. Neuroimage 53:1-15, 2010

11. Farquharson S, Tournier JD, Calamante F, Fabinyi G, Schneider-Kolsky M, Jackson GD, et al: White matter fiber tractography: why we need to move beyond DTI. J Neurosurg 118:1367-1377, 2013

12. Fernandez-Miranda JC, Pathak S, Engh J, Jarbo K, Verstynen T, Yeh FC, et al: High-definition fiber tractography of the human brain: neuroanatomical validation and neurosurgical applications. Neurosurgery 71:430-453, 2012

13. Fiegele T, Feuchtner G, Sohm F, Bauer R, Anton JV, Gotwald T, et al: Accuracy of stereotactic electrode placement in deep brain stimulation by intraoperative computed tomography. Parkinsonism Relat Disord 14:595-599, 2008

14. Frost R, Jezzard P, Douaud G, Clare S, Porter DA, Miller KL: Scan time reduction for readout-segmented EPI using simultaneous multislice acceleration: Diffusion-weighted imaging at 3 and 7 Tesla. Magn Reson Med [epub ahead of print], 2014

15. Gradinaru V, Mogri M, Thompson KR, Henderson JM, Deisseroth K: Optical deconstruction of parkinsonian neural circuitry. Science 324:354-359, 2009

16. Haynes WI, Haber SN: The organization of prefrontal-subthalamic inputs in primates provides an anatomical substrate for both functional specificity and integration: implications for basal ganglia models and deep brain stimulation. J Neurosci 33:4804-4814, 2013

17. Henderson JM: "Connectomic surgery": diffusion tensor imaging (DTI) tractography as a targeting modality for surgical modulation of neural networks. Front Integr Neurosci 6:15, 2012

18. Jenkinson M, Beckmann CF, Behrens TE, Woolrich MW, Smith SM: Fsl. Neuroimage 62:782-790, 2012

19. Jeurissen B, Leemans A, Tournier JD, Jones DK, Sijbers J: Investigating the prevalence of complex fiber configurations in white matter tissue with diffusion magnetic resonance imaging. Hum Brain Mapp 34:2747-2766, 2013

20. Jones DK: Challenges and limitations of quantifying brain connectivity in vivo with diffusion MRI. Imaging Med 2:341-355, 2010

21. Jones DK: Precision and accuracy in diffusion tensor magnetic resonance imaging. Top Magn Reson Imaging 21:8799, 2010

22. Jones DK, Cercignani M: Twenty-five pitfalls in the analysis of diffusion MRI data. NMR Biomed 23:803-820, 2010

23. Jones DK, Knösche TR, Turner R: White matter integrity, 
fiber count, and other fallacies: the do's and don'ts of diffusion MRI. Neuroimage 73:239-254, 2013

24. Keuken MC, Forstmann BU: A probabilistic atlas of the basal ganglia using 7 T MRI. Data Brief 4:577-582, 2015

25. Kristo G, Leemans A, Raemaekers M, Rutten GJ, de Gelder B, Ramsey NF: Reliability of two clinically relevant fiber pathways reconstructed with constrained spherical deconvolution. Magn Reson Med 70:1544-1556, 2013

26. Lambert C, Zrinzo L, Nagy Z, Lutti A, Hariz M, Foltynie $\mathrm{T}$, et al: Confirmation of functional zones within the human subthalamic nucleus: patterns of connectivity and subparcellation using diffusion weighted imaging. Neuroimage 60:83-94, 2012

27. Lambert C, Zrinzo L, Nagy Z, Lutti A, Hariz M, Foltynie T, et al: Do we need to revise the tripartite subdivision hypothesis of the human subthalamic nucleus (STN)? Response to Alkemade and Forstmann. Neuroimage 110:1-2, 2015

28. Leclercq D, Delmaire C, de Champfleur NM, Chiras J, Lehéricy S: Diffusion tractography: methods, validation and applications in patients with neurosurgical lesions. Neurosurg Clin N Am 22:253-268, ix, 2011

29. Little S, Pogosyan A, Neal S, Zavala B, Zrinzo L, Hariz M, et al: Adaptive deep brain stimulation in advanced Parkinson disease. Ann Neurol 74:449-457, 2013

30. Mandelli ML, Berger MS, Bucci M, Berman JI, Amirbekian B, Henry RG: Quantifying accuracy and precision of diffusion MR tractography of the corticospinal tract in brain tumors. J Neurosurg 121:349-358, 2014

31. Marani E, Heida T, Lakke EA, Usunoff KG: The subthalamic nucleus. Part I: development, cytology, topography and connections. Adv Anat Embryol Cell Biol 198:1-113, vii, 2008

32. Milardi D, Gaeta M, Marino S, Arrigo A, Vaccarino G, Mormina E, et al: Basal ganglia network by constrained spherical deconvolution: a possible cortico-pallidal pathway? Mov Disord 30:342-349, 2015

33. Monakow KH, Akert K, Künzle H: Projections of the precentral motor cortex and other cortical areas of the frontal lobe to the subthalamic nucleus in the monkey. Exp Brain Res 33:395-403, 1978

34. Nambu A, Takada M, Inase M, Tokuno H: Dual somatotopical representations in the primate subthalamic nucleus: evidence for ordered but reversed body-map transformations from the primary motor cortex and the supplementary motor area. J Neurosci 16:2671-2683, 1996

35. Nambu A, Tokuno H, Inase M, Takada M: Corticosubthalamic input zones from forelimb representations of the dorsal and ventral divisions of the premotor cortex in the macaque monkey: comparison with the input zones from the primary motor cortex and the supplementary motor area. Neurosci Lett 239:13-16, 1997

36. Østergaard K, Sunde N, Dupont E: Effects of bilateral stimulation of the subthalamic nucleus in patients with severe Parkinson's disease and motor fluctuations. Mov Disord 17:693-700, 2002

37. Parent A, Hazrati LN: Functional anatomy of the basal ganglia. II. The place of subthalamic nucleus and external pallidum in basal ganglia circuitry. Brain Res Brain Res Rev 20:128-154, 1995

38. Parsons TD, Rogers SA, Braaten AJ, Woods SP, Tröster AI: Cognitive sequelae of subthalamic nucleus deep brain stimulation in Parkinson's disease: a meta-analysis. Lancet Neurol 5:578-588, 2006

39. Pollo C, Kaelin-Lang A, Oertel MF, Stieglitz L, Taub E, Fuhr P, et al: Directional deep brain stimulation: an intraoperative double-blind pilot study. Brain 137:2015-2026, 2014

40. Porter DA, Heidemann RM: High resolution diffusionweighted imaging using readout-segmented echo-planar imaging, parallel imaging and a two-dimensional navigatorbased reacquisition. Magn Reson Med 62:468-475, 2009
41. Skare S, Andersson JL: On the effects of gating in diffusion imaging of the brain using single shot EPI. Magn Reson Imaging 19:1125-1128, 2001

42. Sotiropoulos SN, Jbabdi S, Xu J, Andersson JL, Moeller S, Auerbach EJ, et al: Advances in diffusion MRI acquisition and processing in the Human Connectome Project. Neuroimage 80:125-143, 2013

43. Tournier JD, Calamante F, Connelly A: MRtrix: Diffusion tractography in crossing fiber regions. Int J Imaging Syst Technol 22:53-66, 2012

44. Tournier JD, Mori S, Leemans A: Diffusion tensor imaging and beyond. Magn Reson Med 65:1532-1556, 2011

45. Tournier JDD, Yeh CH, Calamante F, Cho KH, Connelly A, Lin CP: Resolving crossing fibres using constrained spherical deconvolution: validation using diffusion-weighted imaging phantom data. Neuroimage 42:617-625, 2008

46. Uğurbil K, Xu J, Auerbach EJ, Moeller S, Vu AT, DuarteCarvajalino JM, et al: Pushing spatial and temporal resolution for functional and diffusion MRI in the Human Connectome Project. Neuroimage 80:80-104, 2013

47. Vo A, Sako W, Niethammer M, Carbon M, Bressman SB, Ulug AM, et al: Thalamocortical connectivity correlates with phenotypic variability in dystonia. Cereb Cortex 25:30863094, 2015

48. Voon V, Kubu C, Krack P, Houeto JL, Tröster AI: Deep brain stimulation: neuropsychological and neuropsychiatric issues. Mov Disord 21 (Suppl 14):S305-S327, 2006

49. Whitmer D, de Solages C, Hill B, Yu H, Henderson JM, Bronte-Stewart H: High frequency deep brain stimulation attenuates subthalamic and cortical rhythms in Parkinson's disease. Front Hum Neurosci 6:155, 2012

50. Wilkins B, Lee N, Gajawelli N, Law M, Leporé N: Fiber estimation and tractography in diffusion MRI: development of simulated brain images and comparison of multi-fiber analysis methods at clinical b-values. Neuroimage 109:341-356, 2015

51. Smith Y, Wichmann T: The cortico-pallidal projection: an additional route for cortical regulation of the basal ganglia circuitry. Mov Disord 30:293-295, 2015

52. Yushkevich PA, Piven J, Hazlett HC, Smith RG, Ho S, Gee JC, et al: User-guided 3D active contour segmentation of anatomical structures: significantly improved efficiency and reliability. Neuroimage 31:1116-1128, 2006

\section{Disclosures}

Dr. Østergaard is a consultant for Medtronic, Inc. She has received personal fees from Medtronic, Inc., outside the submitted work.

\section{Author Contributions}

Conception and design: Petersen, Lund, Rosendal, Østergaard. Acquisition of data: Petersen, Lund, Juul. Analysis and interpretation of data: Petersen, Sunde, Frandsen, Østergaard. Drafting the article: Petersen, Sunde, Frandsen, Rosendal, Juul, Østergaard. Critically revising the article: Petersen, Lund, Østergaard. Reviewed submitted version of manuscript: Petersen, Lund, Sunde, Frandsen, Juul, Østergaard. Approved the final version of the manuscript on behalf of all authors: Petersen. Statistical analysis: Petersen. Administrative/technical/material support: Lund, Frandsen, Rosendal. Study supervision: Østergaard.

\section{Correspondence}

Mikkel V. Petersen, Center of Functionally Integrative Neuroscience, Aarhus University Hospital, Bldg. 10G, 4th Fl., Nørrebrogade 44, 8000 Aarhus C, Denmark. email: mikkel.petersen@ cfin.au.dk. 